\title{
SESSION 6
}

Chairman: C. L. Hemenway 


\title{
31. THE INTERPLANETARY DUST CLOUD
}

\author{
(Survey Paper) \\ T. R. KAISER \\ (University of Sheffield, England)
}

\begin{abstract}
A BSTRACT
The first part of the paper reviews the present state of knowledge of the characteristics of cosmic dust in interplanetary space. Since this is derived from a variety of observational techniques, some attempt is made critically to assess the difficulties in interpretation, particularly those due to differences in observational selection. Attention is drawn to the doubts that recently have arisen concerning the existence of a terrestrial dust cloud. The second part describes some radio investigations of the structure of meteor streams and of the sporadic background. Systematic variations in magnitude distribution with solar longitude which are observed in both the Geminids and Perseids cannot be simply interpreted as due to selective perturbation of the smaller meteoroids. Experimental data are described which point to the existence of considerable radiant structure in the sporadic background.
\end{abstract}

\section{Introduction}

Minor objects in the solar system range in size from the asteroids to dust grains of the order of sub-micron size, which are just large enough to survive the pressures due to solar electromagnetic and corpuscular radiation. The smaller particles have a limited lifetime due to braking effects, and hence we must assume a dynamic equilibrium in which matter is continuously supplied from sources usually believed to be cometary. This is not to say that over the history of the solar system, the cosmic dust cloud has had stationary characteristics; indeed modern views of planetary evolution invoke the prior existence of cosmic dust on a considerable scale. It is perhaps only from studies of material of cosmic origin in deep-sea sediments and, to a limited extent, in tracing backwards orbital parameters obtained from meteors, that we can find direct evidence for evolutionary change in the cosmic-dust distribution.

The range of sizes of the particles we are considering is so wide that no single observational technique encompasses more than a limited part and each is subject to observational selection. Nor are the size distributions obtained in different ways necessarily completely independent estimates. For instance, in interpreting the zodiacal light data it is necessary to postulate the form of the particle-size distribution and this may be obtained from meteor observations, even though these latter refer to much larger particles. Although no single method of observation leads to an unambiguous result for the abundance of cosmic dust, many attempts have been made to assemble and compare the various experimental data. One such is illustrated in 
Figure 1 ; it refers only to the dust at $1 \mathrm{AU}$, and although it presents selected results obtained with the various techniques it is probably fairly representative. Figure 1 attempts to represent only the cumulative size distribution, whereas any complete description must include the distribution of orbital elements (which will also be size-dependent). At the present time this latter is available (subject to observational

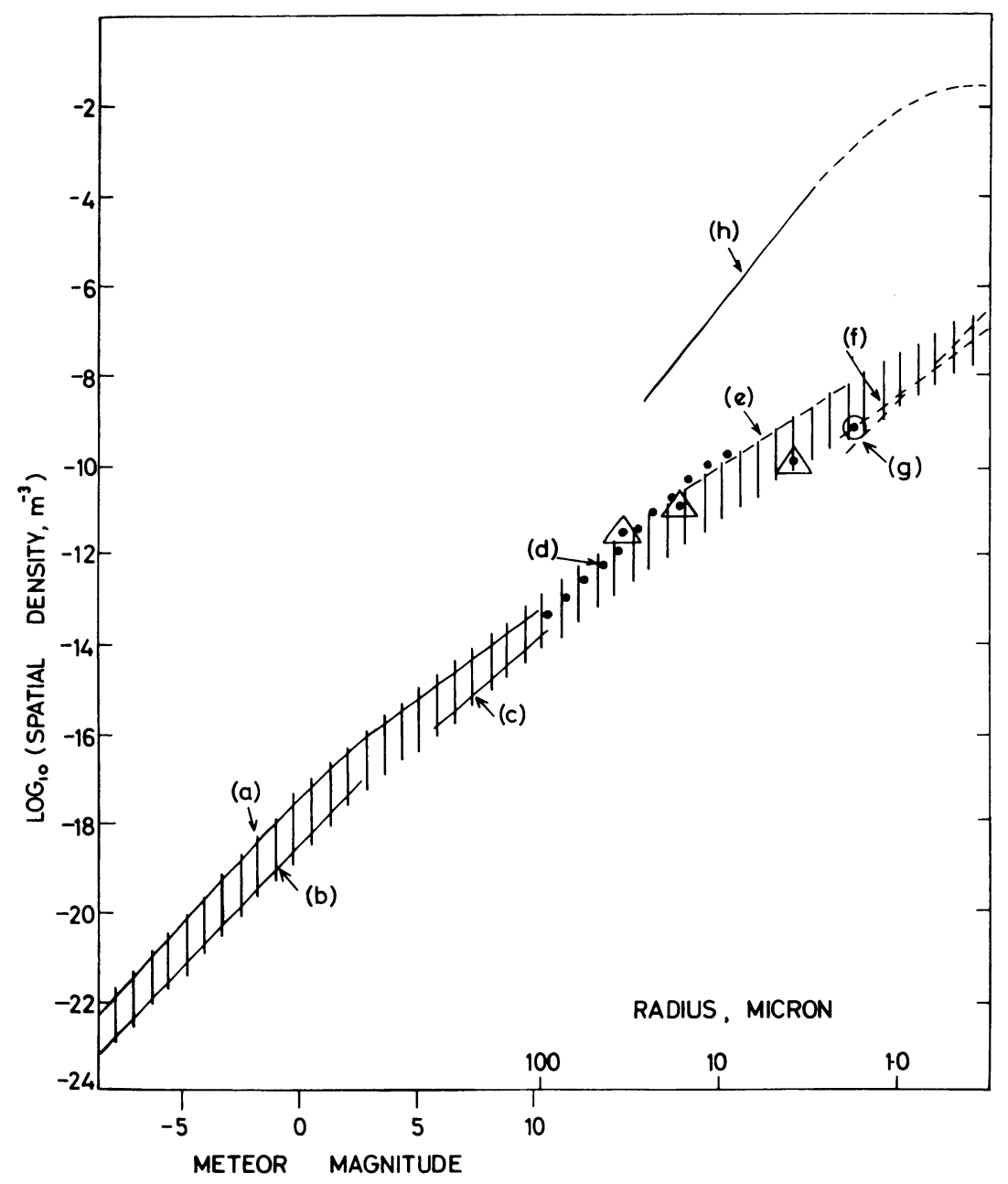

FIG. 1. This represents the number of particles per cubic metre with radii greater than a given value or (in the case of the larger meteoroids) capable of producing meteors brighter than a given magnitude. Sporadic meteor data after (a) Millman (1959); (b) Hawkins and Upton (1958); (c) Kaiser (1961). ..... (d) From accretion of cosmic spherules. -...... Zodiacal-light data after (e) Beard (1959); $(f),(g)$ Ingham (1961) for $t=4$ and $t=5$ respectively. $--(h)$ Near-Earth microphone data (Alexander et al., 1963). $\odot$ Mariner-II microphone data (Alexander, 1962). $\triangle$ Pegasus penetrations (D'Aiutolo et al., 1967). 
selection) only for the larger end of the size spectrum; relatively little even is known of the variation in abundance with distance from the Sun.

The first part of this paper (Section 2) is devoted to a discussion of the information derived from the differing observational methods; the second presents some recent results from radar studies of the structure of meteor streams.

\section{The Interpretation of Observational Data}

All methods of observation lead to an increasing abundance with decreasing particle size (at least down to a few microns) which, over a limited size range, can reasonably be represented by differential and cumulative distribution functions proportional to $r^{-t}$ and $r^{1-t}$ respectively, where $r$ represents the size of particle (radius for a spherical object) and $t$ is a constant.

\subsection{METEORS}

From optical and radio-meteor studies, we can obtain the influx of meteoroids as a function of the luminosity or ionization produced, represented on a logarithmic scale by the magnitude, $M$. Both the differential and cumulative influx can be represented as proportional to $a^{M}$, where $a$ is at most a slowly varying function of $M$; observations lead to values of $a$ ranging between 2.5 and 3.7 for sporadic meteors. The major recurrent meteor showers yield a significant increase in the influx in the brighter visual and photographic meteor range, but the fainter ones tend to be submerged in the sporadic background. Events, such as the great Leonid showers in the 19 th century and 1966, confirm the existence of localized swarms of particles with considerable spatial density, but these are not likely to contribute significantly to the total of interplanetary dust. Assuming the luminosity and ionization produced by a meteoroid to be proportional to its mass, and hence to the cube of its size, the above values of $a$ correspond to values of $t$ between $4 \cdot 0$ and $5 \cdot 3$.

In seeking to derive the size distribution of interplanetary particles from observed meteor rates, there are a number of factors to consider. The simplest in principle is the correction of the observed rates for any variation with magnitude of the proportion of meteors recorded, although this may involve a large and hence relatively uncertain correction for faint radio meteors recorded at such a wavelength that the finite velocities and initial train radii become significant. The conversion of a magnitude distribution into a meteoroid mass or radius distribution involves further difficulties. Ionizing and luminous efficiencies are steeply increasing functions of geocentric velocity leading to observational selection in favour of higher velocities and hence against low-inclination direct orbits (although these may be in the majority). Thus the observed meteor rates tend to be greatest when the elevation of the Earth's apex is a maximum, resulting in a marked diurnal variation due to the Earth's rotation and a 
seasonal one due to the inclination of the Earth's axis, the magnitude of which depends on the latitude of the observer. These effects are further complicated by the fact that the character of the orbits changes with magnitude, such that the fainter telescopic and radio meteors tend to have smaller eccentricity. Thus the mean geocentric velocity will be size-dependent. The observed fragmentation of photographic meteors $(M \lesssim 3)$ has been interpreted in terms of fragile conglomerate particles which makes conversion between magnitude and mass dependent upon assumptions as to particle structure.

The meteor data used in Figure 1 have been reduced on the assumption of compact meteoroids with mean velocity $30 \mathrm{~km} / \mathrm{sec}$ and with radius $0.05 \mathrm{~cm}$ corresponding to 5th magnitude (Kaiser, 1962); in the light of the above, the absolute ordinates can not be regarded as accurate to better than an order of magnitude. An interesting feature is the change in slope at about magnitude +3 . This might be interpreted as a real change in the size distribution if it were not that similar behaviour is exhibited by meteor showers even though they surely are at different stages of evolution. Alternative explanations are either that the smaller meteoroids are compact and the larger conglomerate, or that they are all compact but there is a change in the nature of the physical interaction with the atmosphere. It has been shown (Jones and Kaiser, 1966), for instance, that brittle meteoroids of more than $1 \mathrm{~mm}$ or so in radius (about magnitude +3$)$ are likely to fragment through thermal shock before the commencement of ablation. Recent radio-meteor ablation studies (Poole and Kaiser, 1967) are in support of the view that the fainter meteors are due to non-fragmenting particles. Because of the above possibilities, the radius scale in Figure 1 is restricted to the fainter radio and telescopic meteors.

Meteor observations give details of the structure of the interplanetary dust cloud in the ecliptic plane at $1 \mathrm{AU}$, and some recent observational data are discussed in Section 3. The seasonal variation due to the Earth's orbital motion and the inclination of its axis is opposite in the two hemispheres; however, when the activity is corrected for this and shower activity is subtracted (Kresáková and Kresák, 1955; Weiss, 1957) there remains a true annual variation with a maximum in the latter half of the year (Figure 2).

In 1963, workers in Canada (McIntosh and Millman, 1964) and New Zealand (Ellyett and Keay, 1964) reported an anomalous increase of between 1.5 and 2 times in sporadic meteor activity. These results may be treated with a little caution, since echoes were recorded in range-time coordinates by filming an intensity-modulated cathode ray tube which, in the present author's experience, creates some problems in maintaining constant the effective system sensitivity. Plans are in hand amongst a number of radio-meteor groups to overcome this limitation and extend the scope of the radar technique by digitising the parameters of the radio echoes for computer analysis.

In concluding this section it is worth pointing to the value to be gained from radio- 


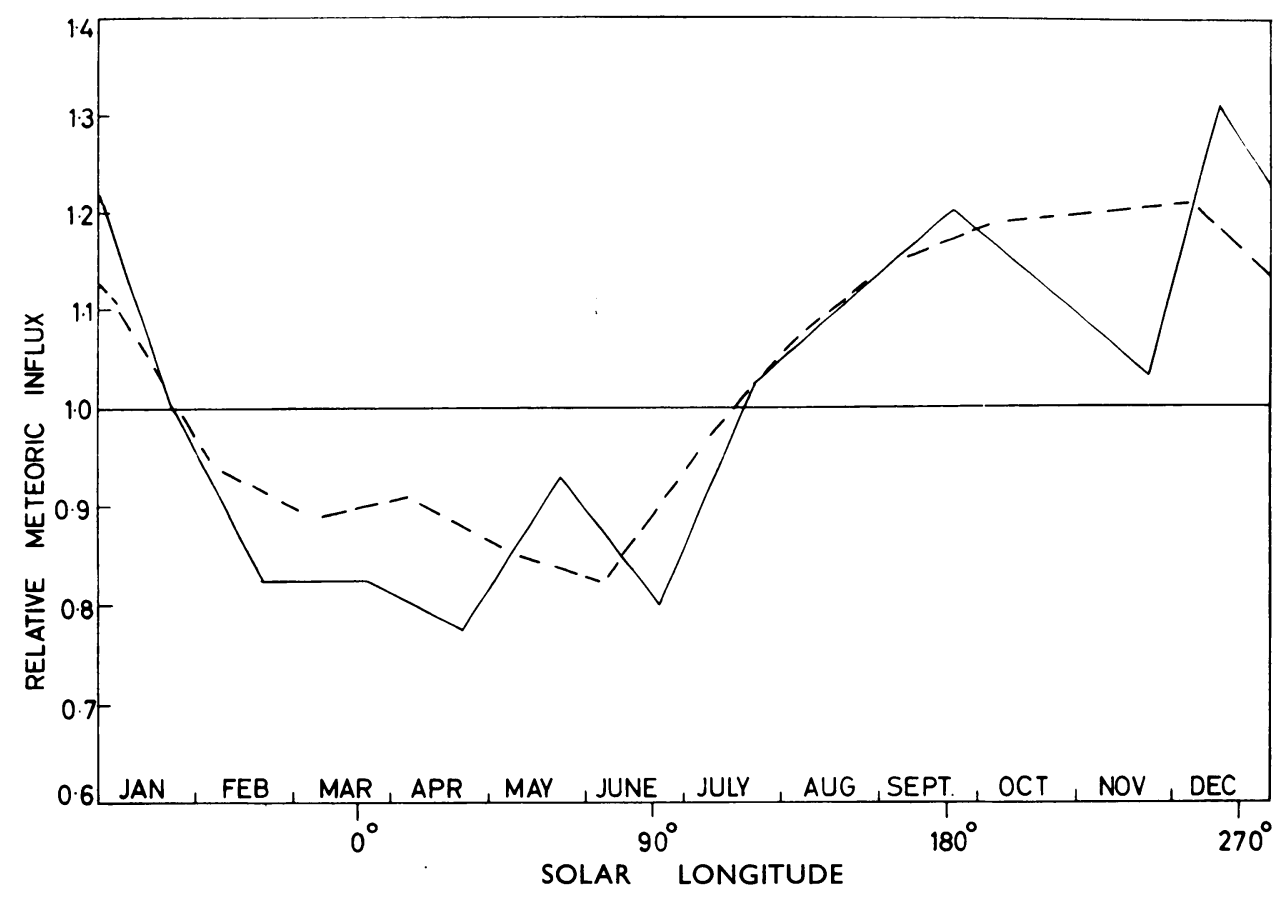

FIG. 2. The annual variation in meteoric activity, corrected for the apex effect. Radio meteors, Southern Hemisphere (Weiss, 1957).-.-.- Telescopic meteors, Northern Hemisphere (Kresáková and Kresák, 1955).

meteor studies made at the equator. It has been shown (Kaiser, 1961) in this case that, for $a \simeq 2.5$ and an aerial beam directed either East or West, the total daily echo rate depends only on the total meteoric influx over the whole Earth per day and is independent of the form of the radiant distribution. This would eliminate the need to correct for the seasonal variation due to the Earth's orbital motion which involves assuming a radiant distribution (Weiss, 1957).

\subsection{TERRESTRIAL ACCRETION}

Interplanetary particles, entering the Earth's atmosphere at cosmic velocities, contribute to accretion at the Earth's surface in several ways. Particles which are sufficiently small (less than a few microns for a velocity of $30 \mathrm{~km} \mathrm{sec}^{-1}$ ) may radiate energy acquired in collisions with air molecules sufficiently rapidly to survive without ablation. Larger meteoroids will also leave some residue when their size and velocity are reduced sufficiently by ablation that radiation loss prevents further ablation. Recent computations (Kaiser and Jones, 1968) show that incident particles which are 
not greatly in excess of the micrometeorite limit nevertheless leave only a small fraction of their initial mass as a residue, hence this source may be neglected. Still larger meteoroids, which fragment before ablation commences, may produce a proportion of particles less than the micrometeorite limit which can then survive. This is only likely to be a significant source of particles less than a few microns, and then only during a major meteor shower.

Surface, deep-sea and ice-core collections have yielded values for the annual dust influx ranging between a few thousand and $5 \times 10^{9}$ tons per year (Kaiser, 1962), which illustrates the great difficulty in identifying the cosmic component and removing terrestrial contamination. Some workers have concentrated, for obvious reasons, on magnetic particles, whereas we may expect a predominance of stony ones. Also, as seen from the preceding paragraph, the atmosphere acts as a size-velocity filter, the effects of which must be allowed for.

In view of the contamination problem, it is tempting to accept the lower figure of a few thousand tons per year (Pettersson and Fredricksson, 1958; Parkin and Hunter, 1962). Parkin and Hunter selected iron and stone spherules, which showed indications of having experienced a temperature during transit through the atmosphere sufficient to cause surface melting; they give both the distribution in size of the particles and the radius of the smallest ones which satisfy their criteria $(7 \cdot 5 \mu$ for iron spherules and, possibly, some 5 times greater for stony ones). If it is assumed that particles less than this limit fail to reach melting point, it is possible to deduce a velocity of entry into the atmosphere which is not greatly in excess of the terrestrial escape velocity, implying direct orbits of low eccentricity (Kaiser, 1962; Öpik, 1956). This may not seem surprising since small particles suffer greater drag due to solar electromagnetic and corpuscular radiation, and their orbits, if initially elliptical, will tend to become circular; it does imply, however, that the initial perihelia of these particles must have been greater than $1 \mathrm{AU}$. It is thus clear that we are dealing with a somewhat different selection of orbits from the meteoroids which produce the optical and radio meteors.

Parkin and Hunter (1962) find $t=4.7$ for the cosmic spherules, and if, with reservations, we accept this as applying also to the interplanetary dust it is possible to estimate, within an order of magnitude or so, the interplanetary abundance (Kaiser, 1962). The result is plotted in Figure 1, assuming 4000 tons per year for accretion of spherules. This joins remarkably well with the meteor results, but for the reasons given above we may be describing significantly different components of the cosmic dust cloud.

We must finally take note of the high values of accretion obtained by a number of workers, when selection of particles is not limited just to those showing evidence of surface melting. A recent novel approach to the accretion problem arises from the detection of radioactive $\mathrm{Al}^{26}$ in deep sea sediments and the conclusion that it is brought in by cosmic dust particles which have experienced solar proton bombard- 
ment. This has led Lal and Venkatavaradan (1966) to estimate a high mass influx of $10^{4}$ tons per day. Singer (1967) has discussed this data and arrives at between 100 and $10^{5}$ tons per day. Although no evidence is available for the sizes of the dust particles responsible, even the lower figure would require an influx much greater than would be deduced by extrapolating the meteor data. Grjebine (1967) has recently reviewed accretion and other data and favours an even higher figure than Singer's upper limit, namely $2 \times 10^{9}$ tons per year.

\subsection{THE ZODIACAL LIGHT}

The scattering of sunlight by interplanetary dust gives rise to the zodiacal light, which may be observed at an elongation from the Sun greater than about $30^{\circ}$, and to a similar phenomenon, the Fraunhofer corona, which can be detected during eclipses out to an elongation of about $15^{\circ}$. The brightness of the F-corona extrapolates relatively smoothly to the zodiacal light (Blackwell et al., 1967), although the scattering in the former case is predominantly through diffraction and, at the greater elongations of the zodiacal light, through reflection. Recent rocket observations (Tanabe and Huruhata, 1967) covering elongations between $15^{\circ}$ and $41^{\circ}$ have filled in the observational gap and are in general agreement with the ground-based data. A part of the scattered light may also be due to free electrons. Important factors which determine the characteristics of the scattered light are: (i) the size distribution and spatial density of the particles and the variation of these quantities through the solar system; (ii) the shape of the particles; (iii) their optical reflecting, refracting and diffracting properties. The polarized component of the light, has been interpreted by some workers as requiring an electron density of a few hundred per cubic centimetre at $1 \mathrm{AU}$ from the Sun, whereas others conclude that the polarization can arise with light scattered from solid particles. Beggs et al. (1964) have studied the profile of Fraunhofer absorption lines and conclude that the electron contribution to the zodiacal light is not greater than a few percent, corresponding to an electron concentration at $1 \mathrm{AU}$ of $16 \pm 20 \mathrm{~cm}^{-3}$. Recent spacecraft measurements of the solar wind (Snyder and Neugebauer, 1963) yield the low value of about $5 \mathrm{~cm}^{-3}$.

It is clear that there are so many parameters involved that various plausible combinations can be made to fit the observations and to yield quite different values for the abundance and size distribution in the dust cloud. A critical parameter is the exponent $t$; if it is relatively large $(\sim 4)$ the scattering from the smaller particles is most important, whereas for smaller values $(\sim 2 \cdot 5)$ the larger particles predominate in the scattering. Depending on which case we choose the estimated spatial density will be sensitive either to the minimum or maximum size of particles present.

Since there are good physical grounds for predicting a minimum size, and since some recent work seems to favour larger values of $t$, we will first consider this case. Ingham (1961) takes the lower limit to the radius as $0 \cdot 3-0.4 \mu$, determined by the 
balance between solar gravitation and radiation pressure, and after investigating the effect of different assumptions arrives at a value of $t$ between 4 and 5. Beard (1959) seeks to allow for solar corpuscular radiation and assumes a minimum radius in excess of $1 \mu$; he also concludes that, unless the particle albedo has an improbably low value, the exponent $t$ must exceed 3 and he adopts the value $3 \cdot 5$. The results of Ingham and Beard are included in Figure 1; it is encouraging that both in magnitude and slope they agree reasonably with extrapolation from meteor data.

The alternative model (low $t$ ) was proposed by Van de Hulst (1947); he obtained $t=2 \cdot 6$, a size range extending to $0 \cdot 1 \mathrm{~cm}$ and a total spatial density several orders of magnitude above the data presented in Figure 1. This is grossly in disagreement, both in magnitude and slope, with the meteor data, even though the size range extends well into the faint telescopic and radio meteor range. The results of Siedentopf (1955) and Elsässer (1963) are in general agreement with Van de Hulst. If this interpretation is correct, we must assume either that the meteor and zodiacal light data refer to quite different selections of particles, or that the sizes estimated for meteoroids are much too low. Nevertheless, Singer (1967) has suggested that the $\mathrm{Al}^{26}$ abundance in deep sea sediments (see Section 2.2) supports the low $t$ hypothesis, which would imply a whole Earth influx of several thousand tons per day (whereas the Ingham and Beard models would lead to a much smaller rate of accretion).

The zodiacal-light data is also capable of yielding information concerning the detailed spatial structure of the dust cloud and the fact that the luminosity is centred on the plane of the ecliptic implies relatively low-inclination orbits. Blackwell et al. (1967) present a model of the dust distribution which requires the density to increase with increasing distance from the Sun out to about $0.5 \mathrm{AU}$, in contradiction with previous models, which supposed an inverse power law with distance with exponent between 1.0 and 1.5 . They find this model, however, to be in contradiction with infrared eclipse observations attributed to thermal emission from dust particles. To reconcile the data they have therefore suggested that the albedo of the particles may decrease towards the Sun by almost two orders of magnitude from $0.5 \mathrm{AU}$, and arrive at an alternative model in which the density decreases with increasing distance from the Sun. Measurements of absorption-line profiles and Doppler shifts can, in principle, yield orbital information, and Ring et al. (1964) have reported a blue shift during evening observations which they interpret as evidence of direct orbits with a velocity of the order of twice the circular value.

Although the zodiacal light is generally attributed to interplanetary dust, satellite evidence for a near Earth concentration (see section 2.5 ) has led to consideration of its possible contribution. Ingham (1962) concludes that at most $10 \%$ of the light results from near-Earth scattering, whereas Divari (1963) finds $10 \%$ to be a lower limit. Doubts which have recently been cast on the satellite data are discussed in section $2 \cdot 5$, and it would help to resolve uncertainties if it proved possible to separate and estimate any near-Earth component in the zodiacal light. 


\subsection{SCATTERING OF LIGHT IN THE ATMOSPHERE}

Small particles which survive ablation will ultimately reach terminal velocity and increase in spatial density as they fall; they may even be supported by upward atmospheric motions. Ground-based observations are made difficult due to molecular scattering; however, one must here mention the pioneer work of Link $(1929,1950)$, who showed that the atmospheric dust concentration will cause diffuseness of the Earth's shadow boundary during eclipses of the Moon. The height discrimination in laser studies is of considerable value in minimising the contribution of molecular scattering; however, the present results are contradictory. Fiocco and Smullin (1963) have reported dust layers above $100 \mathrm{~km}$, whereas Clemeska et al. (1966) have reported a negative result. Noctilucent clouds, which appear at an altitude of about $80 \mathrm{~km}$, are most likely caused by cosmic dust, but until more is known of the dynamic processes which sustain them it is not possible to draw conclusions concerning the influx of dust particles.

\subsection{ROCKET AND SATELLITE OBSERVATIONS}

Spacecraft provide an opportunity for obtaining the most direct evidence both in the vicinity of the Earth and deep into interplanetary space.

The various observational techniques depend upon detecting results of particle impacts such as acoustic impulses, penetration of thin films, pitting of surfaces and luminosity produced on impact, as well as direct collection of particles. Manned satellites have provided the opportunity to expose and recover surfaces which can be examined for evidence of impact and debris which may be deposited. An important parameter in all cases is the total exposure expressed as an area-time product; so far this has limited data to the smaller end of the size spectrum. Rocket measurements suffer particularly in this respect and their interpretation also depends on altitude, i.e. on whether the particles impinge at cosmic velocity or have suffered atmospheric deceleration, in which case they have also been subjected to the atmospheric filter discussed earlier.

The majority of Earth satellite and space-probe studies have used piezo-electric sensors to detect acoustic impulses which have generally been assumed to be proportional to momentum. This is largely based on calibrations in the velocity range $20 \mathrm{~cm}$ $\mathrm{sec}^{-1}$ to $4 \mathrm{~km} \mathrm{sec}^{-1}$ (Dubin, 1960). Recent work (Bohn et al., 1967), which has extended the laboratory measurements into the hypervelocity range up to $10 \mathrm{~km} \mathrm{sec}^{-1}$, suggests that the acoustic impulse is not a linear function of velocity and increases more steeply at hypervelocities $\left(>5 \mathrm{~km} \mathrm{sec}^{-1}\right)$. Various authors have subjected the available data to critical examination and interpretation (see Alexander et al., 1963; Nazarova, 1967; Vedder, 1966). Alexander et al. (1963) and Nazarova (1967) have shown the U.S. and Russian results to be in general agreement and to indicate a much higher 
near-Earth flux, with a larger exponent $t$ than would be deduced from zodiacal-light estimates (on either the 'low $t$ ' or 'high $t$ ' interpretation) or from extrapolated meteor data. The results of Alexander et al. (1963) have been employed (Kaiser, 1962) to deduce the spatial abundance shown in Figure 1. If we use the more recent hypervelocity calibrations (Bohn et al., 1967), the estimated influx and spatial density would be reduced by less than an order of magnitude, still leaving a large gap to be resolved. Deep-space probes, on the other hand, have yielded much lower impact rates. The results of the Venus and Mars probes, Mariners-II and -IV (Alexander, 1962; Alexander et al., 1967) and Mars-I (Nazarova, 1964) are all in closer agreement with zodiacal light and extrapolated meteor data (see Figure 1). Temporary increases in rate of 2-3 orders of magnitude were recorded on Mars-I and attributed to meteor streams, but no similar evidence was obtained with Mariner-IV.

The foregoing results have been interpreted as indicating the presence of a terrestrial dust cloud, either due to concentration of particles in hyperbolic geocentric orbits or to captured particles. Both mechanisms would require particles with low unperturbed geocentric velocities (direct, low-inclination circular orbits), while the latter would give a concentration depending on the dynamic balance set by capture efficiency and lifetime of the captured particles. The effect of drag on captured particles is first to reduce apogee and then to cause them to spiral inwards. The author (1962) has calculated that this would give rise to a spatial density obeying an inverse cube law with radius from the Earth's centre.

Penetration detectors have taken several forms, namely thin-walled pressure cells, photoelectric detection of sunlight admitted through the puncture and thin film capacitors which discharge momentarily when penetrated. These have generally given much lower impact rates than the acoustic detectors, and the large area capacitors on the Pegasus satellites also lead to a flatter slope (lower $t$ ) (D'Aiutolo et al., 1967). The calibration of penetration experiments is probably even more uncertain than that of acoustic devices and the data have been converted to cumulative influx in terms of mass using the crude assumption that the penetration thickness is of the same order as particle diameter. A resulting estimate of spatial density from the Pegasus results, which is given in Figure 1, is hardly likely to be accurate to better than 1 order of magnitude, but this is sufficient to illustrate the disagreement with the microphone data, both in magnitude and slope. The latter appears even flatter than the meteor and zodiacal-light estimates, but in the light of the calibration uncertainty (dependence of penetration on size, density, and velocity) it cannot be accorded too much weight.

Studies of collected particles and cratering on exposed surfaces recovered from rockets and from satellites (Gemini-9 and -12) have tended to give fluxes in some cases in excess even of the acoustic-impulse measurements. There is at present considerable uncertainty in the interpretation of these results (it is not unknown for control surfaces to yield more particles than exposed ones). Of great importance, 
however, is the possibility in collection experiments for studying the physical nature of cosmic dust.

The large difference between near-Earth and deep-space acoustic data, and the apparent agreement of the latter with zodiacal-light and extrapolated meteor measurements, has been widely accepted as incontrovertible evidence for the existence of a terrestrial dust cloud. The low penetration rates from near-Earth satellites has then to be explained. It is true that a different selection of particles must be involved in the two cases, due to different mass and velocity dependence, but it is difficult to see how these alone can be responsible for the discrepancy. A possible explanation is that there are two classes of particles with different physical structure and which differ widely in their capacity for penetration, but, for given momentum, say, produce similar acoustic impulses. Thus we might postulate dense compact particles as responsible for the near-Earth penetrations and the deep-space data and low-density fragile particles for the near-Earth acoustic impulses. The latter would need to be predominant in near-Earth space and of the same order as, or less abundant than compact particles in deep space. This is not inconceivable because, for given mass, such low-density particles have a greater ratio of surface area to volume and hence will be preferentially perturbed (by the Poynting-Robertson effect and the solar wind) into the nearcircular orbits which would be required to produce a significant enhancement near the Earth (in either hyperbolic or closed geocentric orbits). A feature of acoustic impulse observations which is difficult to explain is the reported enhancements of near-Earth impact rate beyond expected statistical fluctuation (Alexander et al., 1963; Dubin et al., 1963), whereas one would expect the characteristics of a terrestrial cloud (of either kind, but especially in the closed orbit case) to be quasi-stationary. Such enhancements have been attributed to meteor streams but here there is even greater difficulty since in these streams the unperturbed geocentric velocity is stach that gravitational concentration will surely be negligible.

Some recent workers, on both theoretical and experimental grounds, have questioned the existence of a near-Earth concentration. In a series of four papers (Shapiro et al., 1966; Colombo et al., 1966a; Colombo et al., 1966b; Lautman et al., 1966) Shapiro and his colleagues have considered several mechanisms for producing near-Earth enhancement, they are: (i) gravitational focussing, (ii) three-body capture (including radiation pressure), (iii) lunar ejection, (iv) air-drag capture. In (iv), radiation pressure is invoked to raise perigee in order to increase the orbital lifeline. They conclude that mechanisms (ii) and (iv) could lead to an enhancement of the order of $10^{2}$ in spatial density at altitude 0.5 Earth radii, but only if a major fraction of the dust has an unperturbed geocentric velocity less than 0.1 and $1.0 \mathrm{~km} \mathrm{sec}^{-1}$ respectively. For a more realistic orbital distribution they conclude that the enhancement must be negligible. Nilsson (1966) has cast doubt on microphone data by showing that the piezo-electric crystals emit noise signals when subject to a slowly varying temperature, and has concluded that the rate of such noise impulses is consistent with flight data 
interpreted as particle impacts. He obtained confirmation for his view from microphone and thin-film capacitor sensors on OGO-II, when the rate of noise impulses increased with the rate of cooling of the microphone sensors. Nilsson explains the so-called 'dust showers' (referred to in the previous paragraph) as due to the same cause, and attributes the low rate of acoustic impulses with deep-space probes to a smaller rate of temperature change. In this case the relative agreement between the deep-space acoustic data and other measurements would be fortuitous.

\subsection{BRIEF SUMMARY}

It is clear from the above that there are considerable problems of data reduction for all methods of observation, largely deriving from lack of knowledge of important parameters, including the nature and structure of particles, the true orbital distribution (which most certainly will not be the same in all size ranges) and hence the distribution of incident velocities upon the Earth or space probe, the relation between ionization, luminosity and mass of meteoroids, the calibration of spacecraft detectors, etc. In particular, the existence of a near-Earth concentration is now subject to doubt. Most of the evidence applies only to the vicinity of the Earth's orbit and even the zodiacal-light measurements do not unambiguously reveal whether the spatial density increases or decreases with distance from the Sun.

There is considerable scope for extension of spacecraft observations. Laboratory generation of hypervelocity particles should enable better calibrations to be made and techniques are already available for measuring the velocity and direction of incidence of particles relative to the vehicle (Nilsson et al., 1965). It is particularly important that the discrepancy between microphone and penetration detectors be resolved.

In view of these problems it is encouraging that, except for the near-Earth acousticimpulse data, it is at least possible to derive a spatial density at $1 \mathrm{AU}$, which, to within the errors involved (at least an order of magnitude), varies relatively smoothly from meteoroids capable of producing the brightest optical meteors to particles approaching the limiting size beyond which radiation pressure must remove them from the solar system; it is represented by the shaded band of Figure 1. There is some discrepancy in the slope of the Pegasus data and it is conceivable that this could be made to extrapolate to the 'low $t$ ' zodiacal-light estimates (although the absolute ordinate is still rather too low); however, as mentioned above, it would be difficult to reconcile this with the meteor results.

\section{The Structure of the Perseid and Geminid Meteor Streams and of the Sporadic Background}

Both the Perseid and Geminid meteor showers recur annually with approximately the same intensity, indicating that material is dispersed around the orbit, and we 
may also expect therefore a dispersal in orbital parameters due to perturbations. In particular, the Poynting-Robertson effect will selectively reduce the aphelia of the smaller particles. Thus, for a shower with low inclination such as the Geminids $\left(i \simeq 23^{\circ}\right)$, the magnitude distribution should vary with solar longitude. As the Earth traverses the stream it should intersect orbits of increasing major axis causing a decrease in the proportion of faint meteors and asymmetry in the curve of activity relative to the maximum. On the other hand, the Perseids have high inclination $\left(i=114^{\circ}\right)$ and we might expect a symmetrical curve in both activity and magnitude distribution.

Systematic radar observations of the major Northern hemisphere meteor showers have been carried out in Sheffield over a period of several years on a frequency of $17 \mathrm{MHz}$. A rhombic aerial was employed, which could be pointed either due West or East. The parameters of the radar were carefully controlled in order to achieve accurate determination of the incident flux and magnitude distribution. Echoes and calibration signals were recorded on magnetic tape which was replayed through a large-screen oscilloscope with a persistent phosphor; thus the echo rates above pre-determined levels of sensitivity were obtained. The reduction of this data to obtain the incident flux and magnitude distribution has been described by Kaiser (1960). The magnitude distribution is obtained from the amplitude distribution of echoes from underdense meteor trains, assumed to be an inverse power law with exponent $s$ (differential distribution) and $s-1$ (cumulative distribution). Assuming maximum ionization to be proportional to meteoroid mass, $s$ is related to the parameters of the magnitude and size distributions by $s=1+2 \cdot 5 \log _{10} a, 3 s=t+2$. For sporadic radio meteors $s$ is generally found to be near to 2 . In the analysis the most sensitive level corresponded to radio magnitude $M=8 \cdot 3$ and $s$ was evaluated over the interval $6 \cdot 8<M<8 \cdot 3$.

\subsection{GEMINIDS}

The main series of observations were conducted in 1962 between November 23 and December 22 (solar longitudes 240-269.5 ); additional observations were made in 1963 and 1964. They have been previously described by Webster et al. (1966). An unexpected feature of the 1962 results was a secondary peak in activity occurring about 1 hour before the expected Geminid peak, which has tentatively been ascribed to a secondary radiant with similar declination to the Geminids but with right ascension about $15^{\circ}$ less. Observations during 1963 and 1964 showed only a slight suggestion of enhanced activity before the expected peak.

The maximum hourly rate of meteors brighter than magnitude $8.3 \mathrm{vs.} \mathrm{solar} \mathrm{longi-}$ tude is shown in Figure 3 for both the true Geminids and the secondary radiant during December 1962 (sporadic activity has been subtracted). The similarity clearly suggests a close association between the two, and leads one to consider the possibility of a perturbation of the parent body before its material was entirely dispersed. 


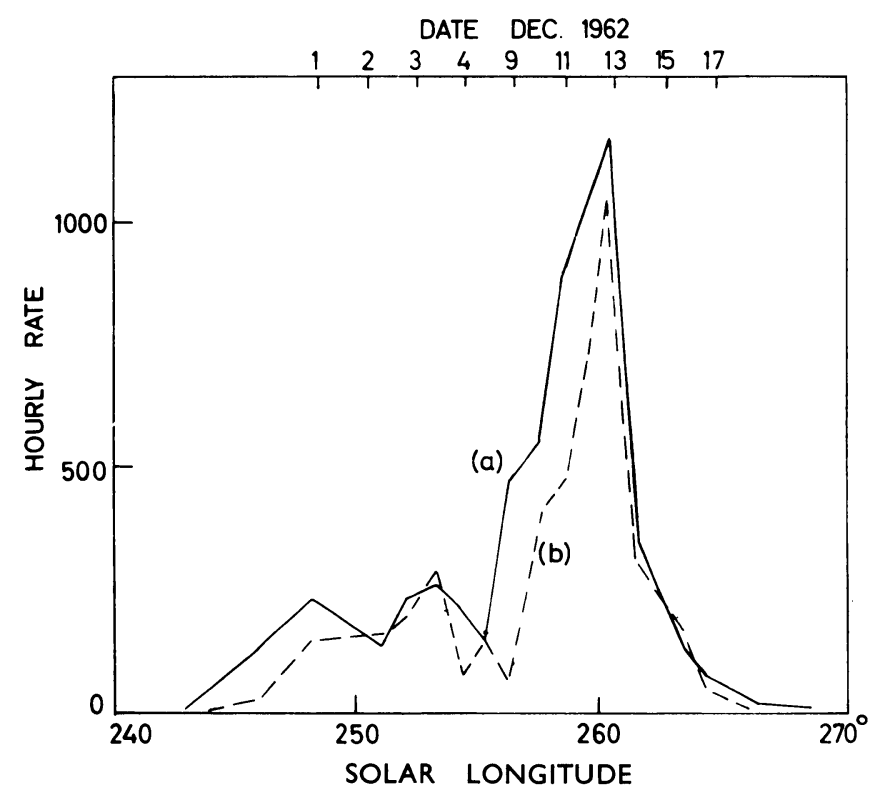

FIG. 3. Maximum hourly rate of shower meteors brighter than magnitude 8.3 vs. solar longitude. (a) Geminids, (b) secondary peak in activity.

The intersection of the two streams at $1 \mathrm{AU}$ and the consequent coincidence in solar longitude of the two centres of activity restricts the choice of the perturbing planet to the Earth, but the maximum deflection suffered by a body with the Geminid geocentric velocity is only about $5^{\circ}$, even for a grazing encounter. Thus for the Earth to have perturbed the orbit of the parent body by a sufficient amount to explain the present results would require multiple encounters. The virtual absence of the secondary activity in 1963 and 1964 suggests that it was due to a periodic stream. It is possibly related to the Sextanid stream, first observed in 1960, which has a similar orbit and which could be a daytime return of a branch of the Geminids. Although observations are rather sparse, a periodicity of 4-5 years has been ascribed to the shower (Weiss, 1960; Nilsson, 1964a). On the other hand, the orbital period of the Geminids is 1.6 years.

The values of the exponent, $s$, are plotted in Figure 4. For the Geminids, there is a clear trend towards lower values with increasing solar longitude, which is in keeping with expectation (due to the Poynting-Robertson effect). There seems to be little indication of a core of large meteoroids near the maximum in activity, as found by Hawkins and Almond (1952). The values of $s$ for the secondary radiant also have a general trend towards smaller values, with a marked minimum at solar longitude $256^{\circ}$, which would seem to indicate a core of large meteoroids at this point.

No velocity measurements were made in 1962; however, studies of the duration 


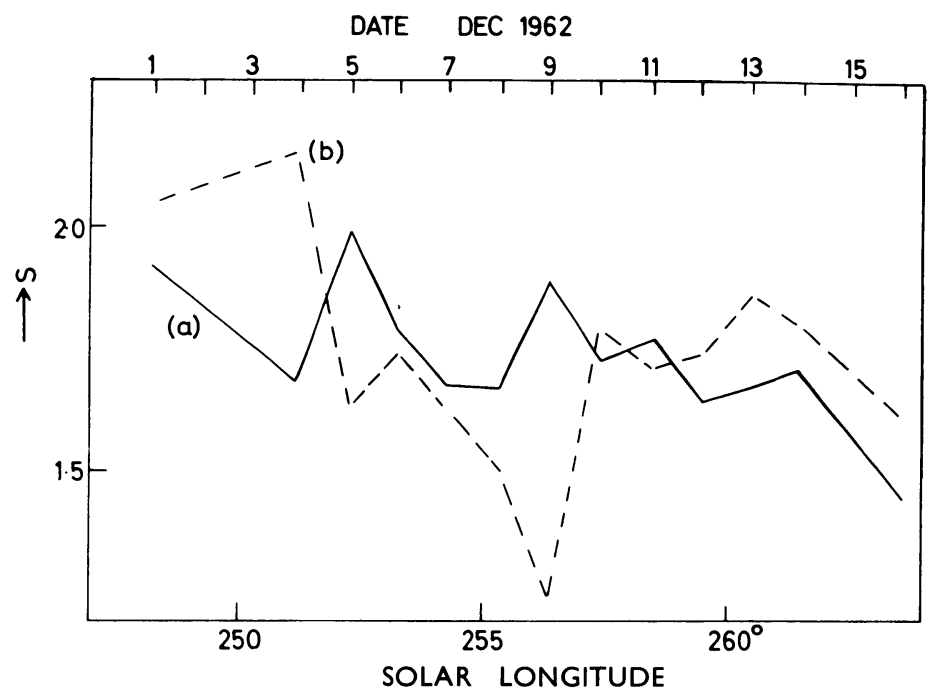

FIG. 4. Variation of the exponent, $s$, with solar longitude. (a) Geminids; (b) secondary radiant.

distributions (Poole and Kaiser, 1967) suggest that the geocentric velocity of meteors from the secondary radiant is close to that of the Geminids.

\subsection{PERSEIDS}

When the hourly rate of Perseids on a given day was compared with the profile expected from the aerial geometry, it was found to peak at the predicted time but the activity clearly extended outside the predicted limits. Examination of range-time records obtained with an intensity modulated display showed a considerable spread in the radiant but indicated also the existence of a relatively discrete centre of activity. By measuring both the maximum rate and the rate outside the time limits predicted for a point radiant, it was possible to estimate separately the contribution from the discrete centre and the diffuse component (Kaiser et al., 1966). These rates are given as a function of solar longitude in Figure 5. It is interesting that the activity from the diffuse component appears to persist to a late date in August, whereas the discrete component is relatively sharply peaked about the maximum.

From the individual daily rate curves, it appears that the angular spread of the radiants in the diffuse components is of the order of $15^{\circ}$. Its existence implies a distribution of orbits which have a common intersection at the Earth's orbit and, again, it is difficult to see how this could come about except by Earth perturbations, in this case of individual meteoroids, but it would clearly require a very large number of encounters to produce the observed structure.

The exponent, $s$, is plotted in Figure 6 for the total activity and separately for the 


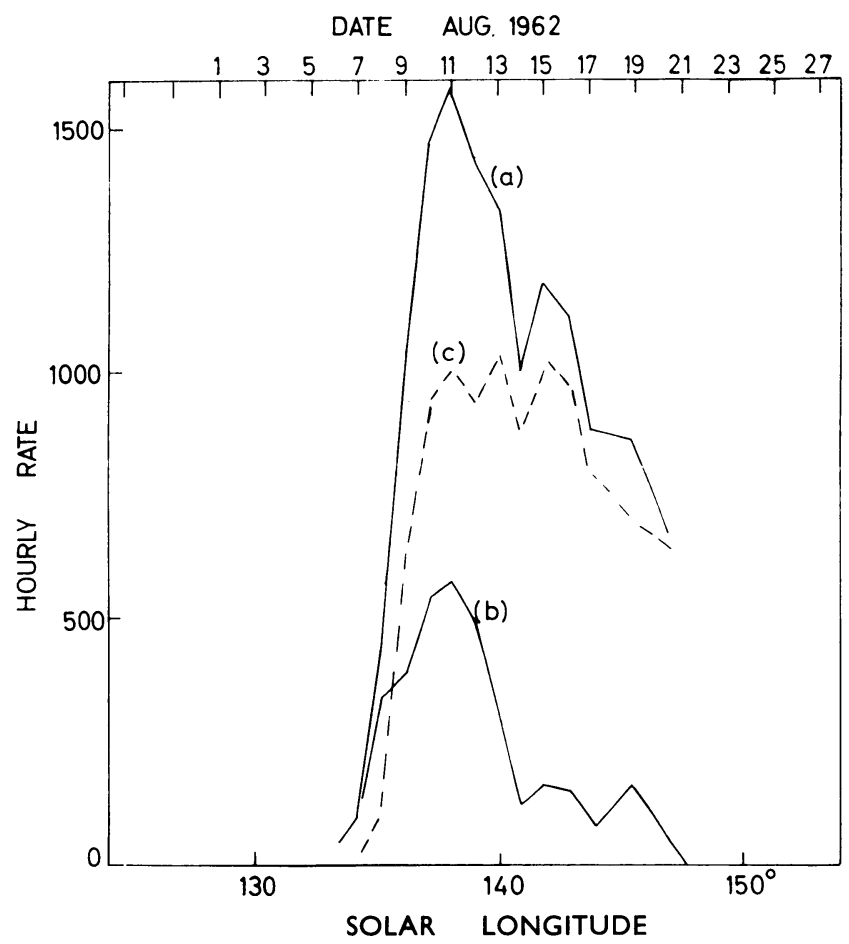

FIG. 5. Hourly rate of Perseid meteors brighter than magnitude 8.3 vs. solar longitude. (a) Total rate; (b) discrete component; (c) diffuse component.

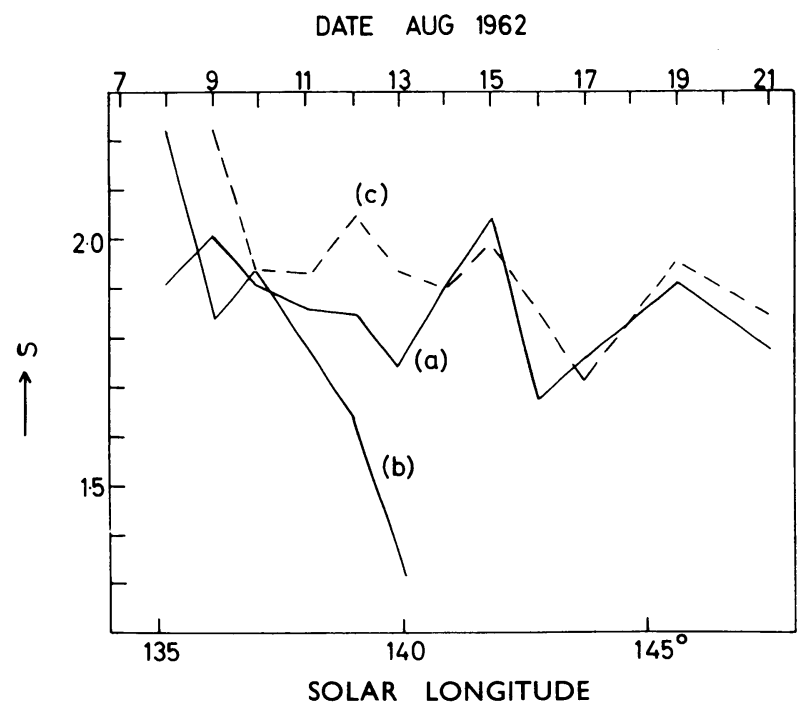

FIG. 6. Variation of $s$ with solar longitude for the Perseids. (a) Total activity; (b) discrete component; (c) diffuse component. 
discrete and diffuse components. There appear to be considerable changes in the magnitude distribution as the Earth traverses the stream, the most striking being the rapid decrease in $s$ for the discrete component (indicating a rising proportion of large meteors). The very low value for the discrete component at solar longitude $140^{\circ}$ is consistent with the idea of a core of large meteoroids, but it does not coincide with the maximum in activity, as suggested by Fedynskij (1959).

Assuming an initially homogeneous distribution, some selective process must be involved to produce these changes in magnitude distribution, but the high inclination appears to rule out the Poynting-Robertson effect. Southworth (1963) considers the effect of planetary perturbations on the history of the stream and concludes that there is evidence for an explosion in the parent body which occurred approximately 500 years ago at a point before perihelion, situated at a distance of $1.5 \mathrm{AU}$ from the Sun and 1.3 AU North of the ecliptic plane. Such an explosion could explain the present width of the Perseid stream at its intersection with the Earth's orbit, and it may be that an explanation for the observed variation in the magnitude distribution is also to be found in such an event.

\subsection{RADIANT STRUCTURE IN THE SPORADIC BACKGROUND}

It is becoming well established that the so-called sporadic background contains a significant proportion of resolvable minor streams. From a random sample of 360 photographic meteors, Hawkins and Southworth (1961) concluded that $12 \%$ belonged to major streams, $13 \%$ to known minor streams and $11 \%$ to new streams. Nilsson (1964b) examined 2200 orbits of radio meteors and finds $25 \%$ to belong to associations which can be said to comprise streams. Lebedinec and Kaščeev (1966) arrived at a similar conclusion from 12500 radio-meteor orbits, $28 \%$ of which could be assigned to a total of 195 meteor streams and associations. What is described in the following is a very simple method for establishing the existence of orbital structure which does not require arbitrary criteria.

During the IQSY a continuous meteor patrol was mounted at Sheffield, using the $17-\mathrm{MHz}$ radar with the rhombic aerial pointing due West. Meteor echoes were recorded in range-time coordinates, using a camera with $35-\mathrm{mm}$ film moving at one inch per hour and an intensity modulated display. The aerial geometry is such that the echo-line of a shower moves outward through the beam and hence the ranges increase as the radiant moves across the sky. All major meteor streams showed range-time movements of the expected kind, but inspection of randomly selected sections of film also gave the impression that there were associations of echoes tending to be grouped along similar contours of increasing range. To overcome the subjectivity of this kind of inspection, the following experiment in pattern recognition was made. Random 4-hour sections of film were printed both directly and in reverse (in the latter case time increased to the left). The prints were shuffled and shown 
individually to a group of subjects who were asked simply to give their first impression as to whether these were associations of echoes (white dots) moving outwards (right-handed) or inwards (left-handed) - 'I don't know' was not accepted as a reply.

In a preliminary experiment every one of 20 subjects identified 6 out of 8 pairs of prints in the manner to be expected if discrete radiant structure was present. In a more comprehensive study, 38 subjects were used with 25 sections of film, each covering a period of several hours, from dates between November 1963 and April 1964 (excluding times of known shower activity) and chosen at random. The result has been analysed statistically by Poole, who concludes that in at least half the samples there is significant activity from one or more unidentified radiants (less than $10 \%$ probability of chance result) while in $25 \%$ the radiant structure is highly developed (less than $1 \%$ probability of a chance result). This is clearly in good agreement with the results of other workers quoted above.

The next step in this kind of analysis would obviously be to seek to identify the associations of echoes using an electronic computer, but the labour involved in digitising the data from films with a meteor rate which may be a thousand or more per hour is prohibitive. For this reason, an electronic on-line digitiser is being developed in Sheffield, which will record parameters (range, amplitude, etc.) of each meteor echo on magnetic tape for computer analysis. This will also make it possible to study fluctuations in magnitude distribution which may provide an additional indicator of associations of meteors.

\section{References}

Alexander, W.M. (1962) Science, 138, 1098.

Alexander, W.M., Berg, O.E., McCracken, C.W., Secretan, L., Bohn, J.L. (1967) Smithson. Contr. Astrophys., 11, 227.

Alexander, W. M., McCracken, C.W., Secretan, L., Berg, O.E. (1963) in Space Res., III, North Holland Publ. Co., Amsterdam, p. 891.

Beard, D. B. (1959) Astrophys. J., 129, 496.

Beggs, D.W., Blackwell, D.E., Dewhirst, D.W., Wolstencroft, R.D. (1964) Mon. Not. R. astr. Soc., $127,329$.

Blackwell, D. E., Ingham, M. F., Petford, A.D. (1967) Mon. Not. R. astr. Soc., 136, 313.

Bohn, J.L., Alexander, W.M., Wever, A. (1967) Reported to the Tenth Meeting of COSPAR, London.

Clemeska, B. R., Kent, G.S., Wright, R.W.H. (1966) Nature, 209, 184.

Colombo, G., Lautman, D. A., Shapiro, I.I. (1966a) J. geophys. Res., 71, 5705.

Colombo, G., Shapiro, I.I., Lautman, D. A. (1966b) J. geophys. Res., 71, 5719.

D'Aiutolo, C.T., Kinard, W.H., Naumann, R.J. (1967) Smithson. Contr. Astrophys., 11, 239.

Divari, N. B. (1963) Astr. Zu., 40, 717.

Dubin, M. (1960) in Space Res., I, North-Holland Publ. Co., Amsterdam, p. 1042.

Dubin, M., Alexander, W.M., Berg, O.E. (1963) Smithson. Contr. Astrophys., 7, 109.

Ellyett, C.D., Keay, C.S.L. (1964) Science, 146, 1458.

Elsässer, H. (1963) Planet. Space Sci., 11, 1015.

Fedynskij, V. (1959) Meteors, Foreign Language Publ. House, Moscow.

Fiocco, G., Smullin, L. D. (1963) Nature, 199, 1275. 
Grjebine, T. (1967) Proceedings of the NATO Adv., Study Inst. on the Mantles of the Earth and Terrestrial Planets, Wiley, London (in press).

Hawkins, G.S., Almond, M. (1952) Mon. Not. R. astr. Soc., 112, 219.

Hawkins, G.S., Southworth, R.B. (1961) Smithson. Contr. Astrophys., 4, 85.

Hawkins, G.S., Upton, E. K.L. (1958) Astrophys. J., 105, 471.

Ingham, M.F. (1961) Mon. Not. R. astr. Soc., 122, 45.

Ingham, M.F. (1962) Space Sci. Rev., 1, 576.

Jones, J., Kaiser, T.R. (1966) Mon. Not. R. astr. Soc., 133, 411.

Kaiser, T.R. (1960) Mon. Not. R. astr. Soc., 121, 284.

Kaiser, T.R. (1961) Mon. Not. R. astr. Soc., 123, 265.

Kaiser, T.R. (1962) Space Sci. Rev., 1, 554.

Kaiser, T.R., Jones, J. (1968) to be published.

Kaiser, T. R., Poole, L. M. G., Webster, A. R. (1966) Mon. Not. R. astr. Soc., 132, 225.

Kresáková, M., Kresák, L. (1955) Contr. Astr. Obs. Skalnaté Pleso, 1, 40.

Lal, D., Venkatavaradan, V.S. (1966) Science, 151, 1381.

Lautman, D. A., Shapiro, I.I., Colombo, G. (1966) J. geophys. Res., 71, 5733.

Lebedinec, V.N., Kaščeev, B.L. (1966) Astr. Zu., 43, 854.

Link, F. (1929) Bull. Obs. Lyon, 11, 229.

Link, F. (1950) Bull. astr. Inst. Csl., $2,59$.

McIntosh, B. A., Millman, P. M. (1964) Science, 146, 1457.

Millman, P. (1959) Can. Aeronaut. J., 5, 358.

Nazarova, T.N. (1964) in Space Res., IV, North-Holland Publ. Co., Amsterdam, p. 921.

Nazarova, T. N. (1967) Smithson. Contr. Astrophys., 11, 231.

Nilsson, C.S. (1964a) Austr. J. Phys., 17, 158.

Nilsson, C.S. (1964b) Austr. J. Phys., 17, 205.

Nilsson, C.S. (1966) Science, 153, 1242.

Nilsson, C.S., Alexander, W.M., McCracken, C.W., Berg, O.E., Secretan, L. (1965) Nature, 208, 673.

Öpik, E.J. (1956) Irish astr. J., 4, 84.

Parkin, D.W., Hunter, W. (1962) Adv. Astr. Astrophys., 1, 105.

Pettersson, H., Fredricksson, K. (1958) Pacific Sci., 12, 71.

Poole, L. M.G., Kaiser, T. R. (1967) Planet. Space Sci., 15, 1131.

Ring, J., Clarke, D., James, J. F., Daehler, M., Mack, J.E. (1964) Nature, 202, 167.

Shapiro, I.I., Lautman, D. A., Colombo, G. (1966) J. geophys. Res., 71, 5695.

Siedentopf, H. (1955) Mém. Soc. R. Sci. Liège, Ser. 4, 15, 96.

Singer, S. F. (1967) Science, 156, 1080.

Snyder, C.W., Neugebauer, M. (1963) J. geophys. Res., 68, 6361.

Southworth, R. B. (1963) Smithson. Contr. Astrophys., 7, 299.

Tanabe, H., Huruhata, M. (1967) Reported to the Tenth Meeting of COSPAR, London.

Van de Hulst, H.C. (1947) Astrophys. J., 105, 471.

Vedder, J.F. (1966) Space Sci. Rev., 6, 365.

Webster, A.R., Kaiser, T.R., Poole, L. M.G. (1966) Mon. Not. R. astr. Soc., 133, 309.

Weiss, A. A. (1957) Austr. J. Phys., 10, 77.

Weiss, A.A. (1960) Mon. Not. R. astr. Soc., 120, 387.

Whipple, F. L. (1961) Nature, 189, 127.

\section{DISCUSSION}

Millman: I wish to make two brief comments. I do not agree with Dr. Kaiser that the method of photographing a cathode ray tube is a major problem in maintaining constant meteor radar rate recording over the year. Although I do not agree entirely with all of Dr. Nilsson's comments on acoustic methods of micrometeoroid detection, I think it was clear from the discussion at the Honolulu symposium in February, 1967, that all acoustic equipment flown on satellites had not necessarily been thoroughly tested for temperature effects in a high-vacuum chamber. 
Lindblad: The anomalous increase in radar rates in 1963 was observed by our group in Sweden, as is evident from a previous paper at this symposium. We used both range-time and amplitude-time radar displays, and only those echoes were counted that were detected on both displays. We therefore feel confident that the increase in 1963 is not an instrumental effect.

Ardeberg: I want to ask a question regarding Figure 6 in your paper. You have not drawn curve $b$, valid for the discrete Perseid component, beyond solar longitude $140^{\circ}$. Is this because you regard the data available beyond $140^{\circ}$ as too sparse to give valid results? Your conclusion that the observed core of large meteoroids does not coincide in solar longitude with the maximum in hourly rates must then be considered as somewhat uncertain, since the observational data in the interval $139^{\circ}-140^{\circ}$ is just above what you regard as worth taking into account.

Kaiser: The ends of the graphs of $s$ vs. solar longitude are where the significance of the values becomes reduced due to the problem of subtracting sporadic meteors and in separating the 'discrete' from the 'diffuse' Perseids. I wish to draw attention to the fact that the variations in $s$ for the Perseids cannot be explained by the Poynting-Robertson effect.

Fedynskij: I would like to comment on the reference to my book which is mentioned by Dr. Kaiser (top, p. 339). There is no contradiction between my statement on the decrease of the value of $s$ during the time of Perseid maximum and the explanation of this fact by Kaiser. The value $s$ diminished indeed near the maximum date of this shower.

Kresák: If the existence of two branches of the Geminid stream is due to perturbations by the Earth, then, according to the Tisserand criterion, the mean geocentric velocity of both branches should be the same. Is this the case in your observations?

Kaiser: From studies of the relation between echo duration and amplitude for both branches of the 1962 Geminids we conclude that the geocentric velocities were the same to within 2 or $3 \mathrm{~km} / \mathrm{sec}$. 